\title{
Die Grotte in Martin Opitz' Schäfferey von der Nimfen Hercinie als Kreuzungspunkt bukolischer Diskurse
}

In der zwölften und letzten Prosa von Iacopo Sannazaros Arcadia gelangt der Erzähler Sincero in ein unterirdisches Höhlensystem, durch das er, geführt von einer Nymphe, aus der arkadischen Welt in seine Heimatstadt Neapel zurückfindet. Die Quellenforschung hat bereits früh darauf hingewiesen, daß die intertextuellen Relationen zwischen Martin Opitz' Schäfferey von der Nimfen Hercinie und Sannazaros Arcadia gerade in der Darstellung des in der antiken und neuzeitlichen Pastoraldichtung äußerst beliebten Motivs der Nymphengrotte besonders eng sind, daß der schlesische Dichter die Beschreibung der Hercinischen Höhle mit Termini durchsetzt, die als wörtliche Übertragungen aus dem italienischen Prätext gelten dürfen. ${ }^{1}$ Die Einsicht, daß Opitz' modellbildende Prosaekloge auf poetische Muster nicht nur aus der italienischen, sondern auch aus der spanischen, französischen und englischen Hirtendichtung rekurriert, sollte nicht den Blick verstellen für die durchaus eigenständige Art und Weise der Aneignung und Funktionalisierung bukolischer Formeln und Motive in der ersten deutschsprachigen Schäfferey. Allein durch die Tatsache, daß Opitz, anders als Sannazaro, die Nymphengrotte vom Rand des Textes in dessen Mitte rückt, gewinnt diese eine Signifikanz, die weit über das hinausgeht, was sich im Rahmen einer nach positivistischen Kriterien verfahrenden Erörterung innerliterarischer Bezüge darlegen ließe. Den zentralen Teil der Schäfferey von der Nimfen Hercinie umfassend, fungiert sie als kohäsionsstiftender Raum, in dem die Opitz' pastorale Dichtung durchwirkenden bukolischen Diskurse sich kreuzen. Einen Beitrag zu deren Erörterung und - nicht nur literarischer - Kontextualisierung will der vorliegende Aufsatz leisten.

Für die Darstellung der Nymphengrotte boten sich nicht nur die von Opitz explizit genannten antiken und frühneuzeitlichen literarischen Vor-

Cf. Alfred Huebner, Das erste deutsche Schäferidyll und seine Quellen, Königsberg 1910, S. 64-86. 
bilder an. ${ }^{2}$ Bedeutsam für die Genese der Schäfferey von der Nimfen Hercinie dürfte in ebenso großem Maße der Einfluß der Grottenarchitektur als Bestandteil aristokratischer Garten- und Schloßanlagen gewesen sein. ${ }^{3}$ Von Italien ausgehend, wo die Gartengrotte in der zweiten Hälfte des 16. Jahrhunderts eine Blütezeit erlebte, verbreiteten sich im 17. Jahrhundert künstliche Höhlen in ganz Europa. Die Grottenanlagen der Villa d'Este in Tivoli, der Medici-Villa in Pratolino, die ১Große Grotter im Boboli-Garten in Florenz, Francesco Primaticcios äußerst kunstvolle Grotte im Schloßpark von Meudon, die mit zahlreichen Automaten bestückten künstlichen Höhlen von St. Germain-en-Laye und schließlich die Thetis-Grotte im Park von Versailles belegen die Relevanz artifizieller Grotten in der Gartenbaukunst der Renaissance und des Barock. In Deutschland fanden künstliche Höhlen erst vergleichsweise spät Eingang in die Parkarchitektur. Auf den Beginn des 17. Jahrhunderts lassen sich die noch heute erhaltenen Grotten des Schlosses Hellbrunn bei Salzburg datieren, wenig später sind diejenigen des Heidelberger Schloßgartens entstanden. Letzteren, »das Hauptbeispiel eines manieristischen Gartens in Deutschland «, wie Wilfried Hansmann urteilt, ${ }^{4}$ kannte Opitz wohl aus eigener Anschauung: 1614 hatte der französische Architekt und Ingenieur Salomon de Caus im Auftrag Friedrichs V. von der Pfalz mit dem Bau des unvollendet gebliebenen und im Dreißigjährigen Krieg fast vollständig zerstörten Hortus Palatinus begonnen, in den mehrere aufwendig ausgestattete artifizielle Höhlen integriert waren. ${ }^{5}$ Als Opitz 1619 nach Heidelberg gelangte, standen die Arbeiten am Schloßgarten kurz

2 Cf. den Hinweis auf die antike Überlieferung sowie auf unmittelbare Vorgänger wie Sannazaro, Castiglione, Gambara, Sidney und d'Urfé in Martin Opitz, Gesammelte Werke, kritische Ausgabe, ed. George Schulz-Behrend, Vol. IV: Die Werke von Ende 1626 bis 1630, Stuttgart 1989f. (= Bibliothek des literarischen Vereins in Stuttgart, Vol. 313) Teil 2, S. 514. Nach dieser Ausgabe (künftig: GW) wird nachfolgend zitiert. Zur literarischen Tradition der Grottendarstellung in Antike und Mittelalter cf. Florence M. Weinberg, The Cave, the Evolution of a Metaphoric Field, from Homer to Ariosto, New York, Bern, Frankfurt a. M. 1986 (= Studies in the Humanities, Literature - Politics - Society, Vol. 4).

3 Zur Grottenarchitektur insbesondere im 16. und 17. Jahrhundert cf. Naomi Miller, Heavenly Caves, Reflections on the Garden Grotto, Boston, London, Sydney 1982, S. 35-76; Barbara Rietzsch, Künstliche Grotten des 16. und 17. Jahrhunderts, Formen der Gestaltung von Außenbau und Innenraum an Beispielen in Italien, Frankreich und Deutschland, München 1987 (= Beiträge zur Kunstwissenschaft, Vol. 17); und Sabine Röder, Höhlenfaszination in der Kunst um 1800, ein Beitrag zur Ikonographie von Klassizismus und Romantik in Deutschland, Remscheid o. J. [1988], S. 39-49.

4 Wilfried Hansmann, Gartenkunst der Renaissance und des Barock, Köln 1983, S. 87.

5 Eine Beschreibung des Heidelberger Schloßgartens enthält Salomon de Caus' Stichwerk Hortvs Palatinvs a Friderico Rege Boemice Electore Palatino Heidelberga exstrvctvs. Ein Nachdruck des Werks ist 1980 erschienen: Salomon de Caus, Die Entwürfe zum Heidelberger Schlossgarten von 1620, Worms 1980. Cf. auch den dazugehörigen Kommentarband von Reinhard Zimmermann, Hortus Palatinus, die Entwürfe zum Heidelberger Schlossgarten von Salomon de Caus, Worms 1986. 
vor dem Abschluß. ${ }^{6}$ Die Darstellung der zur Anlage gehörenden >Großen Grotte in de Caus' 1620 erschienenem Werk Hortvs Palatinvs a Friderico Rege Boemice Electore Palatino Heidelberga exstrvctvs weist bemerkenswerte Ähnlichkeiten mit der Beschreibung der Behausung der Thetis in der Schäfferey von der Nimfen Hercinie auf: Die Ausdehnung der Höhle - es handelt sich um einen »köstlichen saal von großer länge vndt breite « ${ }^{7}-$, die Stein- und Muschelmosaike, die der Beleuchtung dienenden "cristallinnen fenster ${ }^{8}$, "zwey vergüldete altare,${ }^{9}$ das Brunnenbecken, in dem eine "metalline kugel « auf einem Wasserstrahl schwebt, ${ }^{10}$ die in der Mitte der Höhle stehende "lange tafel von polirtem steine ${ }^{11}$ dürften auf de Caus Grottenarchitektur zurückgehen. Dieser hebt im Vorwort seines Stichwerks den großzügigen Grundriß der ’Großen Grotte`, "welche inwendig 70. schuch lang/vnd 32. schuch weit« sei, hervor und weist auf den Reichtum des den Raum auskleidenden Muschelwerks hin. ${ }^{12}$ Die Abbildung der `Großen Grotte` zeigt die einer ausgeklügelten Lichtregie dienenden Fensteröffnungen in der Decke, zwei altarähnliche Konstruktionen im Vordergrund, die Brunnenanlage, mit dem von Opitz beschriebenen Wasserspiel ${ }^{13}$ sowie einen freistehenden Tisch. ${ }^{14}$

Was nun allerdings die durch die Nymphe Hercinie erfahrbar gewordene imaginierte Unterwelt mit den real existierenden künstlichen Höhlen verbindet, sind nicht nur für die Gestaltung des Innenraums konstitutive Elemente wie Wasserspiele, Bauten aus edlen Steinen und Metallen ${ }^{15}$ oder Muschelwerk, ${ }^{16}$ sondern auch und vor allem die mit der künstlichen Grotte

- In der Vorrede zu Hortvs Palatinvs a Friderico Rege Boemia Electore Palatino Heidelberga exstrvctvs betont de Caus, man sei «mit nicht geringer müh vnd Costen/ gegenwertigen Wintermonat dis 1619. Jahrs/ zu vollendung dis gantzen Wercks bey nahe gar gelangt. Vnd wenn die jtzt schwebende Kriegsleuffte nicht darzwischen kommen weren/ hette innerhalb ohngefehr Sechs Monaten alles gantz vnd gar fertig werden mögen« (de Caus, Heidelberger Schlossgarten (Anm. 5) Bl. Ar.). Die in den Futtermauern der Terassen eingebauten Grotten werden im selben Kontext als bereits vollendete beschrieben. Cf. auch Ludwig Schmieder, Der Heidelberger Schloßgarten, eine geschichtliche Studie, in: Mannheimer Geschichtsblätter 37 (1936) 2-56, hier S. 6.

GW IV/2, S. 537, 26.

GW IV/2, S. 538, 7 .

GW IV/2, S. 538, $13 \mathrm{f}$.

Cf. GW IV/2, S. 538, 15-20.

GW IV/2, S. 538, 21.

De Caus, Heidelberger Schlossgarten (Anm. 5) Bl. Aijrf.

13 Brunnenschalen, auf deren senkrechtem Wasserstrahl eine Kugel schwebt, begegnen bei Salomon de Caus gleich mehrfach. Cf. de Caus, Heidelberger Schlossgarten (Anm. 5) no. 19 sowie die in de Caus' 1624 erschienenem Werk Les raisons des forces mouvantes abgebildete Grotte (Elisabeth MacDougall und Naomi Miller, Fons Sapientiae, Garden Fountains in Illustrated Books, Sixteenth-eighteenth Centuries, Washington, D. C. 1977, no. 7).

14 De Caus, Heidelberger Schlossgarten (Anm. 5) no. 22.

is Cf. GW IV/2, S. 539, 1-13.

16 Cf. GW IV/2, S. 536, 23. 
als architektonischem Typus in Zusammenhang stehenden Diskurse. In der Schäfferey von der Nimfen Hercinie begegnen nicht wenige der topoi vorbarocker und barocker Inszenierung artifizieller Höhlenwelten, entscheidender jedoch scheint mir, daß in Opitz' bukolischer Dichtung die der Grottenarchitektur inhärente Problematik des Verhältnisses von Kunst und Natur, von inszeniertem Schein und wirklichem Sein, von aristokratischer Herrschaft und bürgerlichem Hofdienst aufgegriffen und einer komplexen Reflexion unterzogen wird.

Auf vielfältige Weise verbinden sich ars und natura im künstlerischen Konzept der Gartengrotte. Gemeinsam ist den in ihrer Ausgestaltung durchaus divergenten artifiziellen Höhlen des 16. und 17. Jahrhunderts die Verschmelzung künstlicher und natürlicher Elemente, das offensichtliche Bemühen, die Grenzen zwischen göttlicher und menschlicher Schöpfung zu verwischen. Ein meist symmetrischer Grundriß, Fassaden, Säulen, Brunnenbauten verweisen auf die architektonische Grundstruktur der Gartengrotte, natürliche Materialien wie Tuffstein, Muscheln, Korallen, Stalaktiten dienen der Evokation eines Naturraums, der noch nicht dem domestizierenden Zugriff des Menschen ausgesetzt worden ist. Die Synthese von kunstvoller architektonischer Ordnung und natürlicher Dynamik, die in Deutschland im 17. Jahrhundert im Konzept der sala terrena, kulminierte, ${ }^{17}$ generiert ein Ensemble, das materiale Welt auf eine Art und Weise inszeniert, die deren artifiziellen Charakter transparent bleiben läßt. Daß die Gartengrotte sich der Kunstkammer annähern kann, indem sie Bildwerke und Objekte aus den Sammlungen des Auftraggebers integriert, offenbart, in welchem $\mathrm{Maße}$ die sich in der künstlichen Höhle manifestierende Natur die kulturelle Kompetenz des Menschen zum Ausdruck bringt. Wenn das ikonographische Programm wirklicher und fiktiver Grotten sowohl natürliche Tiere als auch Fabelwesen darstellt und Angehörige des Hofs in der Maske mythologischer Figuren auftreten läßt, verschmelzen wirklich erfahrene und mythisch gedachte Welt zu einem Ganzen, innerhalb dessen die Grenzen zwischen natürlicher Kunst und künstlicher Natur fließend sind.

Die kunstvolle Nachahmung natürlicher Phänomene in der Gestaltung artifizieller Grotten wirft die Frage nach dem Verhältnis von inszenierter, scheinhafter und realer Wirklichkeit auf. Seit Plato im 7. Buch des Staats die Höhle als Sinnbild für die Relativität menschlicher Wahrnehmung beschrieben hatte, ist die Perzeption natürlicher und künstlicher Grotten aufs engste mit dem Problembereich des adäquaten Erkennens, beziehungsweise Verfehlens einer sich hinter dem Sichtbaren verbergenden Welt verknüpft. ${ }^{18}$

17 Cf. Elisabeth Herget, Die Sala terrena im deutschen Barock unter besonderer Berücksichtigung ihrer Entwicklung aus der abendländischen Grottenarchitektur, Frankfurt a. M. 1954.

18 Zur Bedeutung der neuplatonischen Höhle in der Kunstgeschichte cf. Naomi Miller, Domain of Illusion: The Grotto in France, in: Fons Sapientiae', Renaissance Garden Fountains, ed. Elisabeth B. MacDougall, Washington D. C. 1978, S. 175-206. 
Angesichts einer technisch immer anspruchsvolleren Ausstattung der artifiziellen Höhlen gewinnt die Frage nach dem Verhältnis zwischen scheinhafter und wirklicher Erfahrung an Bedeutung. Der Fortschritt im Bereich der Naturwissenschaften seit dem 16. Jahrhundert erlaubt die Herstellung zunehmend komplizierter Automaten, die natürliche Phänomene wie Wind, Regen, Donner und Blitz oder Vogelgezwitscher simulieren. Ars und scientia verbinden sich im Versuch, die Illusion eines natürlichen Raums zu schaffen, eine Illusion, die sich vollumfänglich der Kunstfertigkeit technisch begabter Architekten, Ingenieure und Handwerker verdankt. Wenn der Fürst seine Gäste in die Gartengrotte begleitet, sie dem darin inszenierten Naturschauspiel aussetzt, um ihnen anschließend die in Nebenräumen befindlichen der Illusionserzeugung dienenden Maschinen vorzuführen, bewirkt er eine Verwunderung, die aus der ins Bewußtsein der Zuschauer tretenden Diskrepanz zwischen sichtbarer Erscheinung und deren verborgenen Regulationsmechanismen entsteht. Wie auch das für die meisten Grotten kennzeichnende kunstvolle Spiel von Licht und Schatten macht die mechanisch bewerkstelligte Natursimulation die Höhle als Ort erfahrbar, der durch den Wechsel von Verhüllung und Enthüllung zu einer Reflexion über die Möglichkeiten und Grenzen menschlicher Erkenntnis auffordert.

Die Grotte fungiert nicht nur als Schnittstelle zwischen natürlichem und künstlichem Kosmos sowie als Kristallisationspunkt sinnlicher und intellektueller Perzeption, sie fungiert auch als Modell für das Verhältnis zwischen aristokratischer und bürgerlich-gelehrter Welt. Die Kunsthöhle erscheint als Repräsentationsraum adliger Herrschaft, in dem das Wirken des Fürsten auf allegorische Weise zur Darstellung gelangt. Die Fülle an edlen Steinen und Metallen verweist nicht nur auf die Schatzkammer Natur, sie ist zugleich Ausdruck aristokratischen Reichtums, die Wasserströme dienen als Sinnbild fürstlicher abundantia, die die künstliche Natur durchwirkenden normativen Prinzipien stehen mit der ordnenden Macht des Adels in einem Entsprechungsverhältnis. Als Auftraggeberin einer sich an der natürlichen Schöpfung orientierenden artifiziellen Welt, vollzieht adlige Herrschaft eine dem Wirken des Höchsten analoge Handlung, als creator mundi im kleinen gewinnt der Fürst gottähnliche Bedeutung. Die sich in der Grottenarchitektur des 16. und 17. Jahrhunderts manifestierende Glorifizierung aristokratischer Würde ist allerdings nicht denkbar ohne die wissenschaftlichen und künstlerischen Qualitäten meist bürgerlicher Architekten, Ingenieure, Bildhauer und Maler. Indem diese ihre technischen und gestalterischen Fähigkeiten in den Dienst adliger Repräsentation stellen, partizipieren sie am idealtypischen Konstrukt eines Kosmos', in dem kreatürlicher und menschlicher ordo sich in Einklang befinden. Die Symbiose von aristokratischer Herrschaft und bürgerlichem Gelehrten- und Künstlertum bildet die Voraussetzung für die Realisierung eines architektonischen Konzepts, das fürstlichen Absolutismus modellhaft präfiguriert. 
II.

Welche Bedeutung nun kommt den hier knapp skizzierten mit dem Motiv der künstlichen Grotte in Zusammenhang stehenden Diskursen in Opitz' Schäfferey von der Nimfen Hercinie zu? Das Werk setzt ein mit der Beschreibung einer amoenen Landschaft, die als kunstvoll geordneter Naturraum das Verhältnis von ars und natura gleich zu Beginn thematisiert: Die Ausdehnung des Tals, in dem der Erzähler Zuflucht gesucht hat, wird mit mathematischen Termini wie »kreiß« und »halber zirckel« umrissen, »hohe warten«, »dörffer«, »maierhöfe« und »schäffereyen « sind Zeugen menschlicher Zivilisation. Bemerkenswert ist, daß die Gebirgswelt nicht als vom Menschen nachträglich kultivierte Wildnis erscheint, sondern vielmehr als Kosmos, der von der Schöpfung inhärenten Ordnungsprinzipien durchwirkt wird. Auch an anderer Stelle verweist der Text auf den natürlichen ordo, etwa dann, wenn der geregelte Lauf der Gestime ${ }^{19}$ oder der Wechsel der Jahreszeiten $^{20}$ als Beleg für die gesetzmäßige Dynamik der Natur genannt werden. Die kreatürliche Welt bedarf nicht der Domestizierung, um zum locus amoenus zu werden, als artis magistra ist vielmehr sie es, die das Modell für die schöpferische Tätigkeit des Menschen liefert. Die zweimalige Verwendung der Formel »meisterstück der Natur ${ }^{21}$ verweist auf die Meisterin Natur, die als »magdt des Höchsten « $^{22}$ den göttlichen Schöpfungsprinzipien auf exemplarische Weise Gestalt verleiht. Es ist nicht zufällig, wenn die »einöden trawrigen örter ${ }^{23}$ mit dem Teufel in Verbindung gebracht werden und das durch eine Zauberin inszenierte Unwetter - ein Sinnbild des Chaos - zugleich die engen Grenzen des höllischen Machtbereichs markiert. ${ }^{24}$ Geordnete, fruchbare und befriedete Natur als signum göttlichen Waltens verkörpert diejenigen Gesetzmäßigkeiten, denen auch die vom Menschen geschaffenen Werke zu folgen haben. Nachahmung darf demnach nicht einfach auf die Reproduktion sinnlich wahrnehmbarer Erscheinungsformen der Natur zielen, ihr. Bemühen hat sich vielmehr darauf zu richten, das der kreatürlichen Welt inhärente Ordnungsprinzip auch im künstlichen, von Menschenhand geschaffenen Gegenstand erkennbar werden zu lassen. Auch dort, wo sich der künstliche Kosmos scheinbar von der Natur entfernt, bleibt er durch seine Strukturanalogie mit der von Gott geschaffenen Welt Bestandteil der Schöpfung. Aus einer solcherart verstandenen imitatio, aus dem harmonischen Zusammenspiel von natürlicher und menschlicher Kreativität entsteht jene Synthese von natura und ars, die die Hercinische Höhle idealtypisch verkörpert. 
Was Ovid in den Metamorphosen mit Blick auf die Höhle der Thetis festhält, nämlich daß es fraglich sei, »ob Kunst, ob Natur sie geschaffen ${ }^{25}$ gilt auch für die Behausung der Hercinie. Die Grotte, in die die Dichter geleitet werden, ist zwar einerseits als Ursprungsort zahlreicher Flüsse eine natürliche »höle«, zugleich jedoch als Wohnung der Nymphen ein »Erdengemach «, dessen architektonischer Charakter durch das marmorne Eingangstor, den »zirckelrundten « Grundriß und die »cristallenen Säulen « evident wird. ${ }^{26}$ Die Vegetation wird als $» T e p p i c h «$ bezeichnet, ${ }^{27}$ die aus "kleinen muscheln vndt kleinen steinlein ${ }^{28}$ geschaffenen Mosaike wirken kunstvoll wie die Werke des mythischen Malers Apelles. In der »Wohnung ${ }^{29}$ der Thetis schließlich kulminiert die Symbiose von Kunst und Natur in einem Interieur, das sich ganz offensichtlich an den gestalterischen Prinzipien frühneuzeitlicher Grottenarchitektur orientiert. Mosaike, die Fische und "meer wunder ${ }^{30}$ sowie allerlei $» g e f l u ̈ g e l \aleph^{31}$ lebensecht darstellen, Sitzgelegenheiten aus kostbarem Stein, Brunnenbauten, Wasserspiele gehören zu Ausstattung fürstlicher Gartengrotten und bilden hier den Rahmen für ein ikonographisches Programm, das der Verherrlichung der Schaffgotschs dient. Menschliches Handeln, repräsentiert in der Familiengeschichte des schlesischen Adelsgeschlechts, bleibt eingebunden in den fiktional überhöhten Raum eines unterirdischen Saals, in dem Natur und Kultur sich in Einklang befinden. Wenn gegen Ende der Schäfferey die Dichter in Warmbrunn »des glückseligen quelles halben die einheimischen Nimfen« ehren, »des schönen bawes wegen«, mit dem die Quelle umgeben wurde, »aber den Hochwolgebornen vndt werthen Helden Hansen Vlrichen von Schaffgotsch « preisen, wird noch einmal deutlich, welche Bedeutung der harmonischen Verbindung von natürlichem und menschlichem Wirken zukommt.

Nicht nur das Verhältnis zwischen kreatürlicher und vom Menschen geschaffener, sondern auch dasjenige zwischen scheinhafter und seiender Welt erweist sich in der Schäfferey von der Nimfen Hercinie als zentrales Problem. Bereits im einführenden Sonett, in dem der Erzähler die den ersten Teil der Prosaekloge dominierende Thematik der Unvereinbarkeit von Lieben und Reisen dichterisch umreißt, werden Erkenntnis und Blindheit auf konstitutive Weise in Relation gesetzt. ${ }^{32}$ Die besungene abwesende Geliebte, der »Augen trost» des Poeten, ist gekennzeichnet durch das

Ovid, Metamorphosen, übersetzt von Erich Rösch, mit einer Einführung von Niklas Holzberg, München 1990, S. 282 [11, 235]. 
"liecht" ihrer "schönen blicke«, das den Liebenden "verblendt«, sowie durch den "schein« ihres "güldenen haares«. Ihre Ausstrahlung läßt den Dichter blind werden für das »auge der welt die Sonne $«,{ }^{33} »$ mitt gebundenen augen vndt verstopfften ohren ${ }^{34}$ irrt er auf der erfolglosen Suche nach der verlorenen Liebe durch einen Kosmos, der ihm als Exil erscheint. Der Gegensatz von Erkenntnis und Verblendung, von die Sinne betörendem Schein und wahrhaftem Sein durchzieht den gesamten Text: Im perspektivisch gebrochenen, jedoch im wesentlichen neuplatonischen Positionen verpflichteten Gespräch über die Liebe ist von deren wscheinbarem glantze«, unter dem ein "greifflicher betrug « stecke, die Rede; ${ }^{35}$ im Rahmen eines Konzepts, das »der vernunfft vndt liebe vermählung" als Ideal bestimmt, wird die ausschließlich auf die Sinne wirkende vernunftlose Liebe als $"$ schnöde vergengligkeit ${ }^{36}$ denunziert, und diejenige Liebe, die "durch vrtheil vndt verstandt von der außwendigen schönheit zue der inwendigen $\aleph^{37}$ dringt, legitimiert. Die durch die ratio gewährleistete Regulierung der Affekte soll die Unterscheidung zwischen »rechter Schönheit $\aleph^{38}$ und ästhetischem Schein ermöglichen, die die Voraussetzung für die Erkenntnis des Wesens der Liebe bildet. Das Gespräch über Magie, das in der Erzeugung eines künstlichen Unwetters durch eine Zauberin seinen Kulminations- und Endpunkt findet,$^{39}$ die Verurteilung des »blinden wahns« des Volkes, das den in einem petrarkistischen Sonett besungenen Augen der Astree den Wirklichkeitsgrad der Natur zuschreibt, indem es sie für »himmel / sonnen / plitz vndt götter selber « ansieht, ${ }^{40}$ vor allem jedoch der Gang durch die Nymphengrotte verweisen auf das Problem adäquater Erkenntnis. Die platonische Höhle, die den philosophischen Horizont insbesondere des Gesprächs über die Liebe bezeichnet und auf die der Text explizit Bezug nimmt ${ }^{41}$ findet ihre Entsprechung in der Hercinischen Höhle. Anders jedoch als in Platos Höhlengleichnis ist es nicht die Anabasis, der Aufstieg zur Welt des Lichtes, sondern die Erkundung der Nymphengrotte, die Erkenntnis ermöglicht. Der »durch scheinende schleyer«, mit dem Hercinie bekleidet ist, ${ }^{42}$ der $» d u r c h s i c h t i g e ~ g l a n t z «$ der Kristallsäulen, ${ }^{43}$ die Fenster,

GW IV/2, S. 518,5 .

GW IV/2, S. 518, 23.

GW IV/2, S. 523, 5f. Zum Liebesdiskurs in der Schäfferey von der Nimfen Hercinie cf. Peter Rusterholz, Der >Schatten der Wahrheit، der deutschen Schäferdichtung, in: Compara(i)son 2 (1993) 239-259, hier S. 243-251.

GW IV/2, S. 524, 9.

GW IV/2, S. $525,16 \mathrm{f}$.

GW IV/2, S. 523, 27.

Cf. GW IV/2, S. $563,7 \mathrm{ff}$.

GW IV/2, S. 579, $13 \mathrm{f}$.

Cf. GW IV/2, S. 526, 1-13.

GW IV/2, S. 533, 11.

GW IV/2, S. 535, $24 f$. 
durch die der "anmutige tag den gantzen platz von oben her beleuchtete $\ll,{ }^{44}$ stehen zeichenhaft für die Transparenz der von den Nymphen bewohnten Höhlen. Nicht zufällig begegnen im Mittelteil der Schäfferey von der Nimfen Hercinie immer wieder Termini, die optische Wahrnehmung beschreiben: Mit den Worten »So kompt / jhr hirten / schawet an / Was ich / vndt kein mensch zeigen kan« begrüßt Hercinie die Dichter ${ }^{45}$ Nachdem diese die Höhle, in der die Flüsse Schlesiens entspringen, »in augenschein $\aleph^{46}$ genommen haben, werden sie in die Nymphengrotte geführt mit der Aufforderung, "Beschawet nun / [...] das ort / welches für mannes augen zwar sonst verschlossen ist $\ll{ }^{47}$ In der Höhle als Ort der Verborgenheit ${ }^{48}$ erhalten die Dichter Einblick nicht nur in die geologische Beschaffenheit der Erde, sondern auch in die vergangene und zukünftige Geschichte des Adressaten des Hirtengedichts und gelangen so zu einer profunderen Perzeption von Raum und Zeit. Der Eintritt in die Höhle steht - durchaus in neuplatonischem Sinne - am Anfang der visuellen Erfahrung eines verborgenen Kosmos', in dem die Elemente der sichtbaren Welt ihren Ursprung haben; die Anschauung dient als Katalysator eines Erkenntnisprozesses, der im Text wiederholt thematisiert wird.

Klaus Garber hat in seiner Interpretation der Schäfferey von der Nimfen Hercinie darauf hingewiesen, daß Opitz' Prosaekloge aus dem reichhaltigen Fundus an poetischen Möglichkeiten, den die Gattung bereithält, primär die "panegyrische Komponente « realisiert hat. ${ }^{49}$ In der Tat ist das Werk, so der schlesische Autor in der Widmungsvorrede an Hans Ulrich Schaffgotsch, "zue E. Gn. wolverdientem lobe vndt vnsterbligkeit" gedacht. ${ }^{50}$ Der Huldigung an den Adligen dient die den gesamten Mittelteil umfassende Führung durch die unterirdische Welt der Hercinie. Der genealogische Aufriß bildet damit den Angelpunkt des Textes und verweist auf die Bedeutung, die dem Verhältnis zwischen aristokratischer Herrschaft und bürgerlichem Gelehrtentum in Opitz' Schäfferey zukommt. Der der Thetis geweihte Grottensaal versinnbildlicht durch seine natürliche und künstliche Bauelemente amalgamierende Architektur die Konstitutionsprinzipien adliger Herrschaft. Letztere gewinnt ihre Legitimität zum einen aus ihrer Naturhaftigkeit und zum anderen aus ihrer auf Ordnung bedachten Autorität: Die Höhle als Repräsentationsraum der Schaffgotschs ist auch "springkammer

\footnotetext{
GW IV/2, S. 538, 7f.

GW JV/2, S. 534.

GW IV/2, S. 535, $13 \mathrm{f}$.

GW IV/2, S. 535, $17 \mathrm{f}$.

48 Cf. GW IV/2, S. 534, 9 ("verborgene gänge«); GW IV/2, S. 537, 21 (»verborgene gänge des erdtreichs «).

49 Klaus Garber, Martin Opitz' Schäferei von der Nymphe Hercinie, Ursprung der Prosaekloge und des Schäferromans in Deutschland, in: Daphnis 11 (1982) 547603, hier S. 549.
}

so GW IV/2, S. $515,24-$ S. 516,1 . 
der flüße «, ${ }^{51}$ Schatzkammer der Natur und damit Ursprungsort natürlicher Phänomene. Wenn das dynastische Zentrum in den Schoß der Erde verlegt wird, verweist dies auf die naturhafte Genese des schlesischen Adelsgeschlechts. Die kunstvolle Bändigung der Natur wiederum, die sich exemplarisch in den Mosaiken oder den Wasserspielen manifestiert, steht in Entsprechung zum auf Kultivierung bedachten Wirken des Adels. Von den Schaffgotschs wird gesagt, unter ihrer Schirmherrschaft könnten die Nymphen »stiller rhue « genießen ${ }^{52}$ könnten die Bäche ruhig fließen, ${ }^{53}$ könne die Natur in Frieden gedeihen. ${ }^{54}$ Die ordnende Tätigkeit insbesondere Hans Ulrichs äußert sich auch in den von der Familie in Auftrag gegebenen Schloß- und Brunnenbauten, die die Schäfer poetisch würdigen. ${ }^{55}$ Aristokratische Herrschaft erscheint damit als Garantin von Fruchtbarkeit und Frieden, als Fundament einer menschlichen Zivilisationsform, wie sie in der Höhle bildhaft vor Augen geführt wird.

Welche Rolle nun kommt dem sich in den Dienst des adligen Herrn stellenden Gelehrten zu? Als "gedächtniß ${ }^{56}$ bezeichnet Hercinie die Darstellung der Familiengeschichte der Schaffgotschs und weist damit gleich zu Beginn ihrer Ausführungen der memoria eine zentrale Bedeutung zu. Wenn der Nutzen der Historiographie hervorgehoben,${ }^{57}$ die wedelen gemüter sinnreicher poeten «, die ritterliche Taten "in das register der ewigkeit « eintragen, gewürdigt werden, ${ }^{58}$ wenn schließlich in dem die Schäfferey beschließenden Musenanruf die gedächtnisstiftende Funktion von Poesie beschworen wird ${ }^{59}$ macht der Text deutlich, inwiefern künstlerisch-gelehrter Aktivität im Kontext der Legitimation aristokratischer Herrschaft Relevanz zukommt. Ohne das technische und künstlerische Wissen des Gelehrten ist Erinnerung nicht möglich, der Ruhm des Adels stützt sich auf die in kunstvoll geformte Materie übersetzte Glorifizierung aristokratischen Wirkens. Ist der Dichter davon abhängig, daß sein Herr für ein Umfeld sorgt, das der Kunstausübung förderlich ist, so sind die Repräsentanten des Adels auf ein Denkmal angewiesen, das ihren Taten Dauer verleiht. Autorität des Adels und kulturelle Kompetenz des Gelehrtentums bedingen sich gegenseitig; aus der Verbindung von politischem Ordnungsdenken und künstlerischem Formwillen entsteht der überzeitliche Geltung beanspruchende Idealentwurf eines zugleich befriedeten und schönen Kosmos.

GW IV/2, S. 534, 10.

GW IV/2, S. 540, 12.

Cf. GW IV/2, S. 541, $18 f$.

Cf. GW IV/2, S. 546, 13-19.

Cf. GW IV/2, S. 573-578 passim.

GW IV/2, S. 540, 14.

Cf. GW IV/2, S. 541, 10-17.

GW IV/2, S. 547, $12 \mathrm{f}$.

Cf. GW IV/2, S. $577 f$. 
Aus den bisherigen Darlegungen dürfte evident geworden sein, in welchem Maße die Schäfferey von der Nimfen Hercinie auf Diskurse rekurriert, die im Rahmen einer sowohl literarischen als auch architektonischen Wahrnehmung der künstlichen Grotte geradezu topischen Charakter besitzen. Die vorgängig ausgeführte Problematik des Verhältnisses von Kunst und Natur, von ästhetischem Schein und wahrhaftem Sein, von aristokratischer Herrschaft und im Dienst des Hofes stehendem Gelehrtentum durchzieht das gesamte Werk und kulminiert in der Höhle der Hercinie als kohäsionsstiftendem Zentrum eines Textes, dessen formale und motivische Heterogenität ins Auge sticht. In der Nymphengrotte als imaginiertem Raum zwischen künstlicher Natur und natürlicher Kunst werden die verschiedenen Diskurse gebündelt, zueinander in Beziehung gesetzt und damit einer Reflexion unterzogen, die es abschließend zu erörtern gilt.

III.

Auf welche Weise verschiedene, für die bukolische Dichtung konstitutive Diskurse in der Schäfferey von der Nimfen Hercinie verknüpft werden, läßt sich besonders eindrücklich am Beispiel des idealtypischen Modells aristokratischer Herrschaft, das der Text entwirft, nachvollziehen: Als primäre Funktion seines Hirtengedichts hat Opitz in der Widmungsvorrede das Lob Hans Ulrich Schaffgotschs bestimmt. Innerhalb der dreigliedrigen Struktur der Schäfferey von der Nimfen Hercinie nimmt die den gesamten Mittelteil umfassende Panegyrik auf das schlesische Adelsgeschlecht in der Tat einen zentralen Platz ein. Die Gestaltung der Nymphengrotte steht, wie bereits dargelegt wurde, im Dienst der Glorifizierung dynastischer Größe, die kunstvolle Naturszenerie fungiert als Repräsentationsraum aristokratischer Würde.

Die Funktion der Höhlenepisode auf eine »Herrschaftsallegorie legitimistischer Art ${ }^{60}{ }^{6}$ reduzieren zu wollen, greift jedoch zu kurz. Zwar macht der Text die auf die Beglaubigung adliger Herrschaft zielenden Bildformeln pastoraler Dichtung fruchtbar für die Rechtfertigung der den Mitgliedern der Familie Schaffgotsch zukommenden Autorität, die Art und Weise allerdings, in der dies geschieht, offenbart den utopisch-kritischen Anspruch von Opitz' Prosaekloge. Nicht zufällig betont der Dichter in der Widmungsvorrede die Legitimität seiner Panegyrik: der Adressat der Schäfferey

60 Cf. Conrad Wiedemann, Bestrittene Individualität, Beobachtungen zur Funktion der Barockallegorie, in: Formen und Funktionen der Allegorie, Symposion Wolfenbüttel 1978, ed. Walter Haug, Stuttgart 1979 (= Germanistische Symposien, Berichtsbände, Vol. 3) S. 574-591, hier S. 588. 
von der Nimfen Hercinie habe das ihm zugedachte Lob "wolverdient ${ }^{61}{ }^{61}$ Der politische und ökonomische Status des Adligen wird auch an anderer Stelle als »lauteres verdienst der tugendt ${ }^{62}{ }^{2}$ gerühmt. Aristokratisches Wirken erscheint so eingebunden in ein normatives System, dessen Koordinaten sich mit Blick auf die bereits dargelegten mit dem Motiv der künstlichen Grotte verknüpften Diskurse präziser bestimmen lassen.

Solange die Natur als »magdt des höchsten Gottes $\aleph^{63}$ den Maßstab für menschliches Handeln konstituiert, bleibt dieses den religiös fundierten Ordnungprinzipien, die im kreatürlichen Kosmos zum Ausdruck kommen, verpflichtet. Als deus machineus, als Herr über eine künstlich geschaffene Ordnung, ist der Adlige Diener einer ihm übergeordneten Instanz, die seinen Handlungsspielraum bestimmt. Aufgabe des aristokratischen Machthabers ist die Umsetzung der die Natur durchwirkenden Prinzipien in seinem Wirkungsbereich; sein Handeln hat auf die Förderung und den Erhalt einer politisch regulierten, ökonomisch gedeihenden und ästhetisch strukturierten Welt zu zielen. Die Harmonisierung von natürlichem und künstlichem, vom Menschen zu verantwortendem ordo nun setzt die angemessene Wahrnehmung der hinter dem Sichtbaren verborgenen Gesetzmäßigkeiten voraus. Die den Text leitmotivisch durchziehende Spannung von Sein und Schein, von Innen und Außen verweist auf die Bedeutung adäquater Erkenntnis. Letztere manifestiert sich in der Schäfferey von der Nimfen Hercinie unter einer doppelten, sowohl ethisch-moralischen als auch geistigintellektuellen Perspektive: Die Häufung von Begriffen wie »löbliches verhalten $\ll{ }^{64}$ »hohe tugenden $«{ }^{65}$ "guet vndt guetig « ${ }^{66}$ oder aber $» g e s c h i c k l i g-$

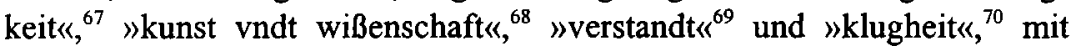
denen die Mitglieder der schlesischen Familie bedacht werden, ist in diesem Kontext bezeichnend. Adel erscheint weniger als die natürliche Folge aristokratischer Herkunft, er ist vielmehr das Ergebnis einer Haltung, die gleichermaßen durch moralische Integrität und intellektuelle Bildung charakterisiert werden kann. Wenn Hans Ulrich Schaffgotschs Kavalierstour als Bildungsreise zu den Stätten humanistischen studiums beschrieben wird, erhebt Opitz' Hirtengedicht die Verbindung von Kunst und Wissenschaft als signum gelehrter Existenz zum Maßstab auch für den Adel. Was der Text mit einer derartigen Argumentation in Gang setzt, ist eine Konvergenzbewegung, deren Fluchtpunkt die Äquivalenz von Blut- und Geistes-

GW IV/2, S. 516,1.

GW IV/2, S. 543, 16.

GW IV/2, S. 539, 12.

GW IV/2, S. 543, 4.

Ibid.

GW IV/2, S. $544,8$.

GW IV/2, S. 545, 22f.

GW IV/2, S. 546, 5.

GW IV/2, S. 546, 6.

GW IV/2, S. 549, 42. 
adel bildet, wie sie in den Worten anklingt "Standt blüet durch verstandt: hett ich nicht standt gehabt / So hette mich verstandt mitt adel doch begabt«. ${ }^{71}$ Damit wird deutlich, daß aristokratische Herrschaft in einem zweifachen Abhängigkeitsverhältnis steht: Als Repräsentantin kreatürlicher Ordnung bleibt sie den in der Natur waltenden Gesetzmäßigkeiten verpflichtet, als Repräsentantin kultureller Ordnung hat sie den Regelsystemen zu folgen, auf denen menschliche Zivilisation basiert. Die Symbiose von adligem Herrn und akademisch gebildetem Hofbeamten ergibt sich aus diesem Angewiesensein auf die im Gelehrten- und Künstlertum verkörperten zivilisatorischen Instrumente. Nur wenn der Hof als Ausdruck und Zentrum aristokratischer Machtbefugnis zur sinn- und ordnungsstiftenden Instanz wird, entgeht er der Gefahr, sich auf ästhetischen Schein zu reduzieren, ein Ort zu sein, "wo rauch vndt schmincke verkaufft wird «. ${ }^{72}$

Was die Schäfferey von der Nimfen Hercinie vollzieht, ist nicht die Apotheose aristokratischer Herrschaft, sondern deren Fundierung in einem normativem System, das den Spielraum aristokratischen Handelns begrenzt. Der herrschaftliche Adel erscheint nicht als Usurpator göttlicher Macht, sondern als Repräsentant einer Ordnung, in die er nicht weniger eingebunden ist als die ihm untergebenen Individuen. Die ihm gesetzten Grenzen veranschaulichen die in bemerkenswerter Häufung auftretenden, der antiken Überlieferung entnommenen exempla für menschliche Hybris, die Verweise also auf die Gigantomachie, ${ }^{73}$ auf die Weberin Arachne ${ }^{74}$ sowie auf den phrygischen König Midas. ${ }^{75}$ Nun gehören Giganten zum ikonographischen Programm einer Vielzahl von Grotten, beispielsweise auch derjenigen von Pratolino bei Florenz. Anders jedoch als Francesco de' Vieri, der in seinem Francesco I. de' Medici, dem Auftraggeber der Villa und der Gärten von Pratolino gewidmeten Werk Delle meravigliose opere di Pratolino e d'Amore (1587) den gescheiterten Aufstand des Riesengeschlechts zeitgenössischer Konvention entsprechend als Warnung vor der Revolte

71 GW IV/2, S. 546, 6f. Die hier beschriebene Konvergenzbewegung steht in Einklang mit dem humanistischen Konzept der nobilitas literaria. Zum Verhältnis von Blutund Geistesadel in der Frühen Neuzeit cf. Volker Sinemus, Poetik und Rhetorik im frülımodernen deutschen Staat, sozialgeschichtliche Bedingungen des Normenwandels im 17. Jahrhundert, Göttingen 1978 (= Palaestra, Vol. 269) S. 207-241; Wilhelm Kühlmann, Gelehrtenrepublik und Fürstenstaat, Entwicklung und Kritik des deutschen Späthumanismus in der Literatur des Barockzeitalters, Tübingen 1982 (=Studien und Texte zur Sozialgeschichte der deutschen Literatur, Vol. 3); Gunter E. Grimm, Literatur und Gelehrtentum in Deutschland, Untersuchungen zum Wandel ihres Verhältnisses vom Humanismus bis zur Frühaufklärung, Tübingen 1983 (= Studien zur deutschen Literatur, Vol. 75); und Joachim Dyck, Ticht-Kunst, deutsche Barockpoetik und rhetorische Tradition, 3., ergänzte Aufl., Tübingen 1991 (= Rhetorik-Forschungen, Vol. 2), S. 113-134.

GW IV/2, S. 532, $15 f$.

Cf. GW IV/2, S. $539,24-540,1$.

Cf. GW IV/2, S. $535,29-536,2$.

75 Cf. GW IV/2, S. 536, 5-18. 
gegen die göttlich autorisierte Macht des Fürsten deutet, ${ }^{76}$ verwendet Opitz in seinem Hirtengedicht die Gigantomachie als Sinnbild für menschliche Anmaßung überhaupt. Die Lehre, »daß die jenigen die sich den himmel an zue tasten vermeßen / von dem himmel verstoßen / vndt von der erden verschlungen werden $«,{ }^{77}$ gilt auch für den Adressaten der Prosaekloge, der mittels einer sich des bukolischen topos der Nymphenhöhle bedienenden Argumentation auf ein Ideal verpflichtet wird, das den in der frühneuzeitlichen Grottenarchitektur zur Darstellung gelangenden Horizont frühabsolutistischer Herrschaftslegitimation sprengt. Die Höhle in Opitz' Schäfferey von der Nimfen Hercinie erscheint so nicht nur als Repräsentationsort, sondern in ebensolchem Maße als Reflexionsraum adliger Macht.

Die erste deutschsprachige Prosaekloge will dem $»$ wolverdienten lobe ${ }^{78}$ eines bedeutenden Repräsentanten des schlesischen Adels dienen und läßt sich damit der panegyrischen Literatur zuordnen; als »lebhafftiger zunder « ${ }^{79}$ der den Adressaten zu derjenigen Haltung ermuntert, die der Text beispielhaft entwirft, steht sie allerdings auch in der Tradition des Fürstenspiegels. Die aus der Verknüpfung schäferlicher Diskurse entstehende Inszenierung genealogisch begründeter Handlungsbefugnis in der Schäfferey von der Nimfen Hercinie verdichtet sich auf kunstvolle Weise zu einem Herrschaftslob und Herrschaftsprogrammatik verknüpfenden idealtypischen Konzept aristokratischer Herrschaft, das auf die Korrespondenz von Kunst und Natur, von äußerer Erscheinung und innerem Sein, von politischer und wissenschaftlicher beziehungsweise künstlerischer Kompetenz zielt.

76 Cf. dazu Élisabeth Mourlot, >Artifice naturek ou snature artificielles: Les grottes médicéennes dans la Florence du XVIe siècle, in: Ville et campagne dans la littérature italienne de la Renaissance, II: Le courtisan travesti, ed. André Rochon, Paris 1977 (= Centre de recherche sur la Renaissance, italienne Vol. 6), S. 303-342, hier S. 329. 